PROCEEDINGS OF THE

AMERICAN MATHEMATICAL SOCIETY

Volume 135, Number 7, July 2007, Pages 2107-2111

S 0002-9939(07)08704-7

Article electronically published on February 6, 2007

\title{
NONCOHERENCE OF SOME RINGS OF FUNCTIONS
}

\author{
AMOL SASANE
}

(Communicated by Joseph A. Ball)

\begin{abstract}
Let $\mathbb{D}, \mathbb{T}$ denote the unit disc and unit circle, respectively, in $\mathbb{C}$, with center 0 . If $S \subset \mathbb{T}$, then let $A_{S}$ denote the set of complex-valued functions defined on $\mathbb{D} \cup S$ that are analytic in $\mathbb{D}$, and continuous and bounded on $\mathbb{D} \cup S$. Then $A_{S}$ is a ring with pointwise addition and multiplication. We prove that if the intersection of $S$ with the set of limit points of $S$ is not empty, then the ring $A_{S}$ is not coherent.
\end{abstract}

\section{INTRODUCTION}

In this paper, we investigate the coherence of some rings of analytic functions. We first recall the notion of coherence.

Definition 1.1. Let $R$ be a commutative ring with identity element $e$, and let $R^{n}=R \times \cdots \times R$ ( $n$ times $)$. Let $f=\left(f_{1}, \ldots, f_{n}\right) \in R^{n}$. An element $\left(g_{1}, \ldots, g_{n}\right) \in$ $R^{n}$ is called a relation on $f$ if $g_{1} f_{1}+\cdots+g_{n} f_{n}=0$. The set of all relations on $f \in R^{n}$, denoted by $f^{\perp}$, is an $R$-submodule of the $R$-module $R^{n}$. The $\operatorname{ring} R$ is called coherent if for each $f \in R^{n}, f^{\perp}$ is finitely generated, that is, there exists a $d \in \mathbb{N}$ and there exist $g_{j} \in f^{\perp}, j \in\{1, \ldots, d\}$, such that for all $g \in f^{\perp}$, there exist $r_{j} \in R, j \in\{1, \ldots, d\}$, such that $g=r_{1} g_{1}+\cdots+r_{d} g_{d}$.

In 5], McVoy and Rubel showed that while $H^{\infty}$ is coherent, the disk algebra $A$ is not coherent. In this paper, we prove the noncoherence of some rings that lie between $A$ and $H^{\infty}$. The rings that we consider are introduced below.

Definition 1.2. Let the open unit disk $\{z \in \mathbb{C}|| z \mid<1\}$ be denoted by $\mathbb{D}$, and the unit circle $\{z \in \mathbb{C}|| z \mid=1\}$ be denoted by $\mathbb{T}$. If $S$ be a subset of $\mathbb{T}$, then

$A_{S}=\{f: \mathbb{D} \cup S \rightarrow \mathbb{C} \mid f$ is analytic in $\mathbb{D}$ and $f$ is continuous and bounded on $\mathbb{D} \cup S\}$, with pointwise addition and multiplication: if $f, g \in A_{S}$, then

$$
(f+g)(z)=f(z)+g(z) \quad \text { and } \quad(f \cdot g)(z)=f(z) g(z), \quad \text { for all } z \in \mathbb{D} \cup S .
$$

Equipped with the supremum norm,

$$
\|f\|_{\infty}=\sup _{z \in \mathbb{D} \cup S}|f(z)| \quad \text { for } f \in A_{S},
$$

the ring $A_{S}$ forms a Banach algebra. We note that if $S=\mathbb{T}$, then $A_{\mathbb{T}}$ is the usual disk algebra $A$, while if $S=\emptyset$, then one obtains the Hardy space $H^{\infty}$.

Received by the editors September 20, 2005 and, in revised form, March 10, 2006.

2000 Mathematics Subject Classification. Primary 46J15, 30A98; Secondary 93C05, 13E15.

Key words and phrases. Banach algebras of analytic functions, coherent rings. 
The spaces $A_{S}$ considered here have been studied earlier (see for instance [1, where among other things, it was shown that the corona theorem holds for $A_{S}$ ). These classes are also of interest in control theory, where they form natural families of irrational transfer functions (see [10, where it was shown that $A_{S}$ is a Hermite domain, although not a Bézout domain, and the stable rank and topological stable rank of $A_{S}$ are 1 and 2, respectively, and the consequences of these properties in control theory were elaborated).

In this note, we study the coherence of the rings $A_{S}$. The relevance of the coherence property in control theory can be found in [7, 8]. For an expository background on coherent rings, we refer the reader to [2]. Noncoherence has been discussed in the context of other rings; for instance, see [6], 4].

Following the ideas of McVoy and Rubel from [5], used for proving the noncoherence of $A$, we show that if $S$ is a subset of the unit circle such that the intersection of $S$ with the set of limit points of $S$ is not empty (that is, $S \cap S^{\prime} \neq \emptyset$, where $S^{\prime}$ denotes the set of limit points of $S$ ), then $A_{S}$ is not coherent. If $S=\emptyset$, then $S \cap S^{\prime}=\emptyset$, and $A_{S}=H^{\infty}$ : in [5], it is shown that $H^{\infty}$ is coherent. In the case when $S$ is not empty and $S \cap S^{\prime}=\emptyset$, we do not know if $A_{S}$ is coherent or not, and this is an open problem.

\section{Noncoherence of $A_{S}$ For $S$ intersecting $S^{\prime}$}

In this section we prove the following.

Theorem 2.1. If $S$ is a subset of $\mathbb{T}$ such that the intersection of $S$ with the set of limit points of $S$ is not empty, then the ring $A_{S}$ is not coherent.

Proof. Let $z_{0}$ be a limit point of $S$ that belongs to $S$, and let $\left(z_{n}\right)_{n \in \mathbb{N}}$ be any sequence in $S \backslash\left\{z_{0}\right\}$ with distinct terms, and with limit $z_{0}$ :

$$
\forall n \in \mathbb{N}, \quad z_{n} \in S \backslash\left\{z_{0}\right\} ; \quad \forall m, n \in \mathbb{N}, \quad z_{m} \neq z_{n} ; \quad \lim _{n \rightarrow \infty} z_{n}=z_{0} \in S .
$$

Let $B_{1}$ be the Blaschke product

$$
B_{1}(z)=\prod_{n=1}^{\infty} \frac{\left|\alpha_{n}\right|}{\alpha_{n}} \frac{\alpha_{n}-z}{1-\alpha_{n}^{*} z}, \quad z \in \mathbb{D},
$$

where

$$
\alpha_{n}=\left(1-\frac{1}{(n+1)^{2}}\right) z_{0}, \quad n \in \mathbb{N} .
$$

Let $B_{2}$ be the Blaschke product

$$
B_{2}(z)=\prod_{n=1}^{\infty} \frac{\left|\beta_{n}\right|}{\beta_{n}} \frac{\beta_{n}-z}{1-\beta_{n}^{*} z}, \quad z \in \mathbb{D},
$$

where

$$
\beta_{n}=\left(1-\frac{1}{\sqrt{2}(n+1)^{2}}\right) z_{0}, \quad n \in \mathbb{N} .
$$

If $m, n \in \mathbb{N}$, then $\alpha_{m} \neq \beta_{n}$ by construction. We also observe that $\left(\alpha_{n}\right)_{n \in \mathbb{N}}$ and $\left(\beta_{n}\right)_{n \in \mathbb{N}}$ are convergent with the same limit $z_{0}$. The functions $B_{1}, B_{2}$ are analytic in an open set $\Omega$ containing $\overline{\mathbb{D}} \backslash\left\{z_{0}\right\}$, and the infinite products converge uniformly on compact subsets contained in $\Omega$ (see for instance Exercise 12 on page 317 of [9]). 
Define $f_{i}: \overline{\mathbb{D}} \rightarrow \mathbb{C}, i \in\{1,2\}$, as follows:

$$
f_{i}(z)=\left\{\begin{array}{cl}
\left(z-z_{0}\right) B_{i}(z) & \text { if } z \in \overline{\mathbb{D}} \backslash\left\{z_{0}\right\} \\
0 & \text { if } z=z_{0}
\end{array}\right.
$$

Then $f_{1}, f_{2} \in A$.

Lemma 2.2. Let $S$ be a subset of $\mathbb{T}$, and let $z_{0} \in S$. Suppose that $f_{i}, i \in\{1,2\}$, are defined by (2.4). If $g_{1}, g_{2} \in A_{S}$ are such that for all $z \in \mathbb{D} \cup S, g_{1}(z) f_{1}(z)+$ $g_{2}(z) f_{2}(z)=0$, then $g_{1}\left(z_{0}\right)=0=g_{2}\left(z_{0}\right)$.

Proof. For all $z \in \mathbb{D}$, we have $\left|g_{1}(z)\right|\left|z-z_{0}\right|\left|B_{1}(z)\right|=\left|g_{2}(z)\right|\left|z-z_{0}\right|\left|B_{2}(z)\right|$, and in particular, $\left|g_{1}\left(\beta_{n}\right)\right|\left|\beta_{n}-z_{0}\right|\left|B_{1}\left(\beta_{n}\right)\right|=0$. As $B_{1}\left(\beta_{n}\right) \neq 0$ and $\left|\beta_{n}-z_{0}\right| \neq 0$, it follows that $g_{1}\left(\beta_{n}\right)=0$. Since $g_{1} \in A_{S}$, we obtain

$$
g_{1}\left(z_{0}\right)=g_{1}\left(\lim _{n \rightarrow \infty} \beta_{n}\right)=\lim _{n \rightarrow \infty} g_{1}\left(\beta_{n}\right)=0 .
$$

Similarly, $g_{2}\left(z_{0}\right)=0$.

We shall prove that the module of relations on $\left(f_{1}, f_{2}\right)$ is not finitely generated. Assume, on the contrary, that the module of relations on $\left(f_{1}, f_{2}\right)$ is generated by $\left(g_{j, 1}, g_{j, 2}\right) \in A_{S}^{2}, j \in\{1, \ldots, d\}$. From Lemma 2.2. it follows that for all $i \in\{1,2\}$ and all $j \in\{1, \ldots, d\}$,

$$
\lim _{n \rightarrow \infty} g_{j, i}\left(z_{n}\right)=g_{j, i}\left(\lim _{n \rightarrow \infty} z_{n}\right)=g_{j, i}\left(z_{0}\right)=0 .
$$

Let $K$ denote the compact set defined as follows: $K=\left\{z_{n} \mid n \in \mathbb{N}\right\} \cup\left\{z_{0}\right\}$. Define the function $\varphi: \mathbb{D} \cup S \rightarrow \mathbb{C}$ by

$$
\varphi(z)=\left(\max \left\{\left|z-z_{0}\right|,\left|g_{1,1}(z)\right|, \ldots,\left|g_{d, 1}(z)\right|,\left|g_{1,2}(z)\right|, \ldots,\left|g_{d, 2}(z)\right|\right\}\right)^{\frac{1}{2}} .
$$

Then it can be seen that the restriction of $\varphi$ to $K$ is a continuous function on $K$. Also, we note that

$$
\forall n \in \mathbb{N}, \quad \varphi\left(z_{n}\right)^{2} \geq\left|z_{n}-z_{0}\right|>0,
$$

and moreover, using (2.5), it follows that

$$
\lim _{n \rightarrow \infty} \varphi\left(z_{n}\right)=0
$$

$K$ is a closed set of Lebesgue measure 0 on the unit circle, and $\varphi: K \rightarrow \mathbb{C}$ is a continuous function on $K$. We now recall the following result (see for instance the theorem on page 81 of $[\underline{3}$ ).

Theorem 2.3 (Rudin). Let $K$ be a closed set of Lebesgue measure zero on the unit circle, and let $F$ be any continuous complex-valued function on $K$. Then there exists a function in the disk algebra $A$ whose restriction to $K$ is $F$.

An application of this theorem yields the existence of a function $\Phi \in A$ whose restriction to $K$ is $\varphi$. Observe that $\Phi\left(z_{0}\right)=\varphi\left(z_{0}\right)=0$. Define $\Gamma_{i}: \mathbb{D} \cup S \rightarrow \mathbb{C}$, $i \in\{1,2\}$, as follows:

$$
\Gamma_{i}(z)=\left\{\begin{array}{cc}
\Phi(z) B_{i}(z) & \text { if } z \neq z_{0} \\
0 & \text { if } z=z_{0}
\end{array}\right.
$$

Then we claim that $\Gamma_{1}, \Gamma_{2} \in A_{S}$, and this can be seen as follows:

Let $i \in\{1,2\}$. As $\Phi$ and $B_{i}$ are analytic in $\mathbb{D}$, it follows that $\Gamma_{i}$ is also analytic in $\mathbb{D}$. 
If $z \in \mathbb{D} \cup S$, then $|\Phi(z)| \leq\|\Phi\|_{\infty}<+\infty$. If $z \in \mathbb{D}$, then $\left|B_{i}(z)\right| \leq 1$. If $z \in S \backslash\left\{z_{0}\right\}$, then each factor in the partial product of (2.2) or (2.3) has modulus 1 , and using the (uniform) convergence of $B_{i}$ on compact subsets contained in $\Omega$, it follows that $\left|B_{i}(z)\right|=1$ for all $z \in S \backslash\left\{z_{0}\right\}$. Consequently $\Gamma_{i}$ is bounded on $\mathbb{D} \cup S$.

Finally, continuity of $\Gamma_{i}$ on $(\mathbb{D} \cup S) \backslash\left\{z_{0}\right\}$ follows from the continuity of $B_{i}$ and that of $\Phi$ on $(\mathbb{D} \cup S) \backslash\left\{z_{0}\right\}$. Continuity of $\Gamma_{i}$ at $z_{0}$ follows by observing that it is the product of a bounded function and the continuous function $\Phi$ which converges to 0 . So we conclude that $\Gamma_{1}, \Gamma_{2} \in A_{S}$.

Let $g_{1}:=\Gamma_{2}$ and $g_{2}:=-\Gamma_{1}$. Then $\left(g_{1}, g_{2}\right)$ is a relation on $\left(f_{1}, f_{2}\right)$. Indeed, if $z \in(\mathbb{D} \cup S) \backslash\left\{z_{0}\right\}$, then

$g_{1}(z) f_{1}(z)+g_{2}(z) f_{2}(z)=\Phi(z) B_{2}(z)\left(z-z_{0}\right) B_{1}(z)-\Phi(z) B_{1}(z)\left(z-z_{0}\right) B_{2}(z)=0$. If $z=z_{0}$, then $g_{1}(z) f_{1}(z)+g_{2}(z) f_{2}(z)=0 \cdot 0+0 \cdot 0=0$.

However, we now show that there cannot exist functions $h_{1}, \ldots, h_{d}$ in $A_{S}$ such that

$$
h_{1}\left(g_{1,1}, g_{1,2}\right)+\cdots+h_{d}\left(g_{1, d}, g_{2, d}\right)=\left(g_{1}, g_{2}\right) .
$$

If (2.8) holds for some $h_{1}, \ldots, h_{d}$ in $A_{S}$, then for all $n \in \mathbb{N}$,

$$
\begin{aligned}
& h_{1}\left(z_{n}\right) g_{1,1}\left(z_{n}\right)+\cdots+h_{d}\left(z_{n}\right) g_{d, 1}\left(z_{n}\right)=g_{1}\left(z_{n}\right)=\Gamma_{2}\left(z_{n}\right) \\
& =\Phi\left(z_{n}\right) B_{2}\left(z_{n}\right)=\varphi\left(z_{n}\right) B_{2}\left(z_{n}\right) .
\end{aligned}
$$

Hence for all $n \in \mathbb{N}$, we have

$$
\begin{aligned}
\left|\varphi\left(z_{n}\right)\right| & =\left|\varphi\left(z_{n}\right) B_{2}\left(z_{n}\right)\right| \leq\left|h_{1}\left(z_{n}\right)\right|\left|g_{1,1}\left(z_{n}\right)\right|+\cdots+\left|h_{d}\left(z_{n}\right)\right|\left|g_{d, 1}\left(z_{n}\right)\right| \\
& \leq d \cdot \max \left\{\left\|h_{1}\right\|_{\infty}, \ldots,\left\|h_{d}\right\|_{\infty}\right\} \cdot \max \left\{\left|g_{1,1}\left(z_{n}\right)\right|, \ldots,\left|g_{d, 1}\left(z_{n}\right)\right|\right\} \\
& \leq M \cdot \max \left\{\left|z_{n}-z_{0}\right|,\left|g_{1,1}\left(z_{n}\right)\right|, \ldots,\left|g_{d, 1}\left(z_{n}\right)\right|,\left|g_{1,2}\left(z_{n}\right)\right|, \ldots,\left|g_{d, 2}\left(z_{n}\right)\right|\right\} \\
& =M \cdot\left|\varphi\left(z_{n}\right)\right|^{2},
\end{aligned}
$$

where $M:=d \cdot \max \left\{\left\|h_{1}\right\|_{\infty}, \ldots,\left\|h_{d}\right\|_{\infty}\right\}$. Consequently, using (2.6), we have

$$
\forall n \in \mathbb{N}, \quad 1 \leq M\left|\varphi\left(z_{n}\right)\right| \text {. }
$$

But from (2.7), the right-hand side of (2.9) tends to 0 as $n \rightarrow \infty$, and so we arrive at the contradiction that $1 \leq 0$. This completes the proof.

\section{REFERENCES}

1. J. Détraz. Étude du spectre d'algèbres de fonctions analytiques sur le disque unité. (French) Comptes Rendus Hebdomadaires des Séances de l'Académie des Sciences. Séries A et B, 269:A833-A835, 1969. MR0254604 (40:7812)

2. S. Glaz. Commutative coherent rings: historical perspective and current developments. Nieuw Archief voor Wiskunde, nos. 1-2, 4:37-56, 1992. MR.1187898 (93j:13024)

3. K. Hoffmann. Banach Spaces of Analytic Functions. Dover, 1988. MR1102893 (92d:46066)

4. M. Jerman. When is $C(X)$ a po-coherent ring? Communications in Algebra, no. 4, 30:19491959, 2002. MR.1894053 (2003b:06018)

5. W.S. McVoy and L.A. Rubel. Coherence of some rings of functions. Journal of Functional Analysis, 21:76-87, 1976. MR0410393 (53:14143)

6. C.W. Neville. When is $C(X)$ a coherent ring? Proceedings of the American Mathematical Society, no. 2, 110:505-508, 1990. MR0943797 (90m:54024)

7. A. Quadrat. The fractional representation approach to synthesis problems: an algebraic analysis viewpoint. Part I: (Weakly) doubly coprime factorizations. SIAM Journal on Control and Optimization, 42:266-299, 2004. MR1982745 (2004f:93062)

8. A. Quadrat. The fractional representation approach to synthesis problems: an algebraic analysis viewpoint. Part II: Internal Stabilization. SIAM Journal on Control and Optimization, 42:300-320, 2004. MR1982746(2004f:93063) 
9. W. Rudin. Real and Complex Analysis. 3rd Edition, McGraw-Hill, 1987. MR0924157 (88k:00002)

10. A.J. Sasane. Irrational transfer function classes, coprime factorization and stabilization. CDAM Research Report CDAM-LSE-2005-10, May 2005. Available electronically at http://www.cdam.1se.ac.uk/Reports/Files/cdam-2005-10.pdf

Department of Mathematics, London School of Economics, Houghton Street, LonDon WC2A 2AE, United Kingdom

E-mail address: A.J.Sasane@lse.ac.uk 